\title{
The clinical characteristics of Chinese Takayasu's arteritis patients: a retrospective study of 411 patients over 24 years
}

\author{
Jing Li, Fei Sun, Zhe Chen, Yunjiao Yang, Jiuliang Zhao, Mengtao Li, Xinping Tian ${ }^{*}$ and Xiaofeng Zeng ${ }^{*}$
}

\begin{abstract}
Background: We aimed to investigate the clinical characteristics of 411 Chinese Takayasu's arteritis (TAK) patients using a retrospective analysis.

Methods: We retrospectively reviewed 810 medical charts of patients with a diagnosis of TAK who were admitted to Peking Union Medical College Hospital from 1990 to 2014. 411 patients with a complete dataset were finally included in the analysis. The demographic data, clinical features, angiographic patterns, and TAK-related surgical procedures were collected and analyzed.

Results: The median age at disease onset was $23(18,30)$ years old, with a median disease duration of $21(6,60)$ months; 325 (79.1\%) were female. The angiographic involvement pattern was type I in 91 (22.1\%) patients, type Ila in 16 (3.9\%) patients, type IIb in 16 (3.9\%) patients, type III in 12 (2.9\%) patients, type IV in 26 (6.3\%) patients, and type $V$ in $250(60.8 \%)$ patients. Subclavian arteries (79.8\%) were the most commonly involved, followed by carotid arteries (79.1\%). The occurrence rate (4.1\%) of aortic aneurysm in this study was low; 119 operations and interventions were performed. The most common cause of death in this study was heart failure.

Conclusion: Subclavian arteries, carotid arteries, and type $\vee$ were the most frequently involved arteries and angiography pattern in this Chinese TAK study. The difference in angiographic features may lead to differences in clinical manifestations. Surgical operation and interventions should be performed at different stages of the disease course.
\end{abstract}

Keywords: Takayasu's arteritis, Clinical characteristics, Angiography manifestation, Carotid artery, Aneurysm

\section{Background}

Takayasu's arteritis (TAK) is an uncommon systemic inflammatory vasculitis with granulomatous inflammation in the adventitia and media of the aorta and its major branches [1]. The estimated annual incidence of TAK is 0.4-1.0 per million people in Germany [2], 2.0 per million in southeast Norway [3], and 3.4 per million in the northwestern part of Turkey [4]. In the UK primary care cohort, the prevalence is only 4.7 per million [5], but it is more common in southeast Asia, India, Japan, and Mexico [4]. The prevalence of TAK was estimated to be 40 per million in Japan [6]. In an autopsy series

\footnotetext{
*Correspondence: tianxp6@126.com; xiaofeng.zeng@cstar.org.cn Department of Rheumatology and Clinical Immunology, Peking Union Medical College Hospital, Peking Union Medical College and Chinese Academy of Medical Sciences, Key Laboratory of Rheumatology and Clinical Immunology, Ministry of Education, Beijing 100032, China
}

from Japan, the prevalence was reported to be as high as 1 in 3000 cases [7]. In the northwestern part of Turkey, the prevalence (33 per million) of TAK is higher than that of the western population (4.7-8.0 per million) [5, 8-10], but is similar to east Asia [4].

TAK mainly involves the aorta and its major branches. Fibrosis develops gradually as the disease progresses, followed by stenosis or occlusion of the vessels, which in turn results in organ ischemia [11]. Occasionally, the destruction of the elastica and muscularis may result in artery dilation or aneurysm [11]. The prevalence of aneurysm in Southern African patients [12], Thai patients [13], and Chinese patients [14, 15] seems to be very different. The angiographic patterns also vary in different ethnic groups [16]. In this study, we investigated the angiographic involvement patterns, clinical 
features, and the outcome of Chinese TAK patients, and also compared our data with the different ethnic groups reported in the literature.

\section{Methods \\ Patients}

We retrospectively reviewed 810 medical charts of patients with a diagnosis of TAK who were admitted to Peking Union Medical College Hospital (PUMCH), a Chinese national referral center, from 1990 to 2014. Charts of the same patients for multiple admissions were considered as a single case. Thus, finally, 411 patients with a complete dataset were included in this study, and the angiography manifestations of each patient with the most extended blood vessels involved were analyzed as some patients had more than one angiography evaluation performed during follow-up.

\section{Methods}

A case search was performed electronically via the information systems of our hospital using The International Classification of Diseases Tenth Revision (ICD-10) code for TAK (M31.4). The diagnosis was confirmed through a chart review by two senior rheumatologists according to the 1990 American College of Rheumatology (ACR) criteria for TAK [17]. The information for these patients during their admission(s) in our center was collected. A database file was built under the guidance of one senior rheumatologist according to the information needs of this study. The data were entered into the database by four junior rheumatologists using Epidata (Version 3.1), and data were exported to SPSS software for further analysis. The input data in the database were mutual checked by all four rheumatologists to ensure their accuracy and completeness.

The demographic data, clinical presentations, physical examination findings, laboratory tests, angiographic involvement patterns, interventions, and surgical procedures were collected and analyzed. Renal dysfunction was defined as estimated glomerular filtration rate (eGFR) $<90 \mathrm{ml} / \mathrm{min} / 1.73 \mathrm{~m}^{2}$. Heart failure was defined as ejection fraction $\leqq 40 \%$ by echocardiography or typical clinical symptoms (i.e., circulatory congestion, exertional dyspnea, orthopnea, etc.) with rales heard on a physical examination, with or without pleural effusion seen on chest radiography [18]. The angiographic involvement pattern was based on the 1994 Numano et al. criteria [19], and the description records of angiography were analyzed. Each patient had blood vessel examinations either by catheter angiography or computed tomography angiography (CTA) at least once to assess the extent of blood vessel involvement. If patients had symptoms that suggested possible intra-cranial, pulmonary, or coronary vessel involvement, then CTA or magnetic resonance angiography (MRA) for intra-cranial, pulmonary, or coronary arteries were performed. This is the general protocol for all inpatients with TAK. However, this may underestimate the prevalence of the special vessel involvement if the vessel change is not severe enough to cause clinical symptoms.

We searched PubMed with the keywords "Takayasu's arteritis" and "cohort study" to obtain angiographic manifestations of other ethnic groups. The inclusion criteria for patients in these studies were all based on the 1990 ACR criteria for TAK, and patient numbers in studies over 50 were included in this study. Comparisons were made between this study and the studies we obtained from the literature.

\section{Statistical analysis}

Because some of the data were not distributed in a normal pattern, we described the numerical variables as median (Q1, Q3), and the categorical variables as number (percentage). Comparisons between different populations were made using Chi-square tests for categorical data. Fisher's exact tests were conducted when the expected frequencies were less than 5. A two-sided $P$ value less than 0.05 was considered to be statistically significant. Analysis was performed with the SPSS software (version 19.0, IBM SPSS statistics, Armonk, New York, USA).

\section{Results}

\section{Demographic data and clinical manifestations}

The male to female ratio of this study was 1:3.8 (325 female, 86 male), with the median age of disease onset being 23 years old and the median duration of disease from onset 21 months. The clinical features and angiographic classification are presented in Table 1. Type $\mathrm{V}$ (60.8\%) was the most common angiographic involvement pattern. Fever (31.1\%) was the most common constitutional symptom, followed by malaise (29.7\%) and weight loss (20.0\%). Claudication, vessel bruits, and other symptoms/signs due to deficiency of arterial supply were common. Heart lesions and renal involvement due to hypertension were observed in this group of patients. The median levels of erythrocyte sedimentation rate (ESR), C-reactive protein (CRP), and high-sensitive CRP (hs-CRP) were all elevated (Table 1).

\section{Features of vessel involvement and distributions}

The detailed distribution of vessel involvement is shown in Table 2. Among them, subclavian arteries (79.8\%) and carotid arteries $(79.1 \%)$ were the most commonly involved arteries, almost at the same frequency.

We found another six cohort studies from different geographic areas [12, 20-24] which also described the details of involved vessels. The occurrence rate of 
Table 1 Demographic data and clinical features of 411 Chinese TAK patients

\begin{tabular}{|c|c|}
\hline & Patient number \\
\hline \multicolumn{2}{|l|}{ Gender } \\
\hline Female & $325(79.1 \%)$ \\
\hline Male & $86(20.9 \%)$ \\
\hline Age at onset (years) & $23.0(18.0,30.0)^{a}$ \\
\hline Duration of disease at first admission (months) & $21.0(6.0,60.0)^{\mathrm{a}}$ \\
\hline \multicolumn{2}{|l|}{ Constitutional findings } \\
\hline Fever & $128(31.1 \%)$ \\
\hline Malaise & $122(29.7 \%)$ \\
\hline Weight loss & $82(20.0 \%)$ \\
\hline \multicolumn{2}{|l|}{ Symptoms } \\
\hline Arthralgia & $37(9.0 \%)$ \\
\hline Skin rash & $32(7.8 \%)$ \\
\hline \multicolumn{2}{|l|}{ Vascular findings } \\
\hline Bruit of carotid arteries & $257(62.5 \%)$ \\
\hline Hypertension & $209(50.9 \%)$ \\
\hline Asymmetric pulsation & $158(24.6 \%)$ \\
\hline \multicolumn{2}{|l|}{ Claudication } \\
\hline Upper limbs & $60(14.6 \%)$ \\
\hline Lower limbs & $57(13.9 \%)$ \\
\hline Gangrene & $2(0.5 \%)$ \\
\hline \multicolumn{2}{|l|}{ Cardiac findings } \\
\hline Valvar lesions & $201(48.9 \%)$ \\
\hline Left ventricle hypertrophy on ECG & $58(14.4 \%)$ \\
\hline Heart failure & $61(14.8 \%)$ \\
\hline Pericardial effusion & $30(7.3 \%)$ \\
\hline Myocardial lesions & $26(6.3 \%)$ \\
\hline Angina & $14(3.4 \%)$ \\
\hline Myocardial infarction & $3(0.7 \%)$ \\
\hline \multicolumn{2}{|l|}{ Renal abnormalities } \\
\hline Nephrotic-range proteinuria & $13(3.2 \%)$ \\
\hline Renal dysfunction & $47(11.4 \%)$ \\
\hline \multicolumn{2}{|l|}{ Neurological symptoms } \\
\hline Headache & $125(30.4 \%)$ \\
\hline Ischemic stroke & $22(5.4 \%)$ \\
\hline Syncope & 49 (11.9\%) \\
\hline \multicolumn{2}{|l|}{ Ophthalmoscope findings } \\
\hline Hypertensive retinopathy & $31 / 165^{b}(18.8 \%)$ \\
\hline TAK-related retinopathy & $23 / 165^{b}(13.9 \%)$ \\
\hline Ischemic changes & $11 / 165^{b}(6.7 \%)$ \\
\hline Vasculitis presentations & $11 / 165^{b}(6.7 \%)$ \\
\hline Others & $3 / 165^{\mathrm{b}}(1.8 \%)$ \\
\hline
\end{tabular}

Table 1 Demographic data and clinical features of 411 Chinese TAK patients (Continued)

\begin{tabular}{|c|c|}
\hline \multicolumn{2}{|l|}{ Angiographic classification } \\
\hline Type I & $91(22.1 \%)$ \\
\hline Type lla & $16(3.9 \%)$ \\
\hline Type Ilb & $16(3.9 \%)$ \\
\hline Type III & $12(2.9 \%)$ \\
\hline Type IV & $26(6.3 \%)$ \\
\hline Type V & $250(60.8 \%)$ \\
\hline \multicolumn{2}{|l|}{ Laboratory findings } \\
\hline Level of ESR (mm/h) & $26.0(11.0,65.0)^{a}$ \\
\hline Level of CRP (mg/L) & $12.8(3.4,44.8)^{a}$ \\
\hline Level of hs-CRP (mg/L) & $8.8(2.0,12.5)^{\mathrm{a}}$ \\
\hline Level of WBC $\left(10^{9} / \mathrm{L}\right)$ & $8.2(6.6,11.0)^{a}$ \\
\hline Level of Hgb (g/L) & $121(108,135)^{a}$ \\
\hline
\end{tabular}

carotid artery and subclavian artery involvement was similarly high, but the occurrence rates of aortic involvement and aortic aneurysm were different (Table 3).

Ten studies from 11 different ethnic groups were found $[13,15,16,25-28]$, which also described the angiographic involvement pattern based on the classification of Numano et al. [19]. In eight groups, type V was the most common pattern, as seen in the present study, but in the other three groups, type I was the most common pattern (Table 4).

\section{TAK-related surgical procedures and interventions}

In this study, 119 operations and interventions were performed at different stages of disease, including 54 (13.1\%) bypass surgery, 25 (6.1\%) percutaneous transluminal angiography (PTA) for renal artery stenosis or occlusion, $22(5.4 \%)$ PTA for stenosis or occlusion in other arteries, 35 (8.5\%) stent implantation for stenosis, and $11(2.7 \%)$ other operations for TAK-related lesions, including five valve replacements, four nephrectomies, and two repairs of aortic aneurysm (Table 5).

\section{Mortality rate and causes of death}

In this study, 12 patients deceased at a median age of $33.5(19.8,59.8)$ years old. The median survival time was 102.5 (46.0, 242.5) months. The direct causes of death were heart failure in five, bleeding in two, pulmonary infection in two, sudden cardiac death due to severe pulmonary hypertension in one, postoperative complication in one, and end-stage malignancy in one patient [29]. 
Table 2 The manifestations of vessels involved in 411 Chinese Takayasu's arteritis patients

\begin{tabular}{|c|c|c|c|c|c|}
\hline Arteries & Any arterial lesion & Stenosis & Occlusion & Dilatation & Aneurysm \\
\hline Subclavian artery & $328(79.8 \%)$ & $232(56.4 \%)$ & $130(31.6 \%)$ & $11(2.7 \%)$ & $5(1.2 \%)$ \\
\hline Left & $283(68.9 \%)$ & 185 (45.0\%) & $110(26.8 \%)$ & $4(1.0 \%)$ & $3(0.7 \%)$ \\
\hline Right & $223(54.2 \%)$ & 145 (35.3\%) & $58(14.1 \%)$ & $8(1.9 \%)$ & $3(0.7 \%)$ \\
\hline Carotid artery & 325 (79.1\%) & 241 (58.6\%) & $102(24.8 \%)$ & $16(3.9 \%)$ & $3(0.7 \%)$ \\
\hline Left common & 291 (70.8\%) & 175 (42.6\%) & $82(20.0 \%)$ & $6(1.5 \%)$ & 0 \\
\hline Right common & $266(64.7 \%)$ & 156 (38.0\%) & 53 (12.9\%) & $9(2.2 \%)$ & $3(0.7 \%)$ \\
\hline Left internal & 85 (20.7\%) & $56(13.6 \%)$ & 19 (4.6\%) & $5(1.2 \%)$ & 0 \\
\hline Right internal & 94 (22.9\%) & $61(14.8 \%)$ & $15(3.6 \%)$ & 7 (1.7\%) & 0 \\
\hline Left external & 59 (14.4\%) & $36(8.8 \%)$ & $14(3.4 \%)$ & $2(0.5 \%)$ & 0 \\
\hline Right external & 55 (13.4\%) & 38 (9.2\%) & $9(2.2 \%)$ & $1(0.2 \%)$ & 0 \\
\hline Renal artery & 201 (48.9\%) & 182 (44.3\%) & $43(10.5 \%)$ & $6(1.5 \%)$ & $2(0.5 \%)$ \\
\hline Left & $157(38.2 \%)$ & 143 (34.8\%) & $24(5.8 \%)$ & $5(1.2 \%)$ & $1(0.2 \%)$ \\
\hline Right & $149(36.3 \%)$ & $133(32.4 \%)$ & $24(5.8 \%)$ & $1(0.2 \%)$ & $1(0.2 \%)$ \\
\hline Abdominal aorta & $158(38.4 \%)$ & $134(32.6 \%)$ & 12 (2.9\%) & $16(3.9 \%)$ & $10(2.4 \%)$ \\
\hline Vertebral artery & $118(28.7 \%)$ & $82(20.0 \%)$ & 46 (11.2\%) & $10(2.4 \%)$ & $1(0.2 \%)$ \\
\hline Left & 94 (22.9\%) & $62(15.1 \%)$ & 34 (8.3\%) & $6(1.5 \%)$ & $1(0.2 \%)$ \\
\hline Right & 81 (19.7\%) & 55 (13.4\%) & $23(5.6 \%)$ & $10(2.4 \%)$ & 0 \\
\hline Mesenteric artery & 116 (29.7\%) & $90(21.9 \%)$ & $33(8.0 \%)$ & $1(0.2 \%)$ & 0 \\
\hline Thoracic aorta & 86 (17.5\%) & 72 (17.5\%) & 0 & $14(3.4 \%)$ & $3(0.7 \%)$ \\
\hline Innominate artery & 81 (19.7\%) & $61(14.8 \%)$ & $16(3.9 \%)$ & $8(1.9 \%)$ & $1(0.2 \%)$ \\
\hline Iliacofemoral artery & 65 (15.8\%) & $43(10.5 \%)$ & $18(4.4 \%)$ & 0 & $4(1.0 \%)$ \\
\hline Left & $53(12.9 \%)$ & $32(7.8 \%)$ & $15(3.6 \%)$ & 0 & $3(0.7 \%)$ \\
\hline Right & $54(13.1 \%)$ & $36(8.8 \%)$ & $11(2.7 \%)$ & 0 & $2(0.5 \%)$ \\
\hline Ascending aorta & $39(9.5 \%)$ & $4(1.0 \%)$ & 0 & 37 (9.0\%) & $3(0.7 \%)$ \\
\hline Aortic arch & $32(7.8 \%)$ & $25(6.1 \%)$ & 0 & $7(1.7 \%)$ & $1(0.2 \%)$ \\
\hline Intracranial artery ${ }^{a}$ & $31(7.5 \%)$ & $29(7.1 \%)$ & $2(0.5 \%)$ & 0 & 0 \\
\hline Pulmonary artery ${ }^{b}$ & $31(7.5 \%)$ & 17 (4.1\%) & $14(3.4 \%)$ & $1(0.2 \%)$ & 0 \\
\hline Left & $22(5.4 \%)$ & $12(2.9 \%)$ & $6(1.5 \%)$ & $1(0.2 \%)$ & 0 \\
\hline Right & $27(6.6 \%)$ & $12(2.9 \%)$ & $11(2.7 \%)$ & $1(0.2 \%)$ & 0 \\
\hline Coronary artery $^{c}$ & $15(3.6 \%)$ & $14(3.4 \%)$ & $4(1.0 \%)$ & 0 & 0 \\
\hline Left & $13(3.2 \%)$ & $10(2.4 \%)$ & $3(0.7 \%)$ & 0 & 0 \\
\hline Right & 7 (1.7\%) & 7 (1.7\%) & $1(0.2 \%)$ & 0 & 0 \\
\hline
\end{tabular}

${ }^{a} 131$ patients with imaging of intracranial artery

${ }^{\mathrm{b}} 45$ patients with imaging of pulmonary arteries

${ }^{\mathrm{c}} 42$ patients with imaging of coronary arteries

\section{Discussion}

As a rare systemic large vessel vasculitis, most reported studies in the literature on TAK are small in sample size. In China, the prevalence of TAK is relative higher than many other geographic areas, such as Europe and North America. In this study, we retrospectively analyzed the data of 411 inpatients with complete angiographic description. According to our knowledge, this is the largest series of patients with TAK who were admitted to a rheumatology department thus far.
TAK can be divided into two phases, the acute phase and the chronic or stenotic phase, based on clinical presentations. The acute phase, which is characterized by nonspecific constitutional symptoms caused by acute inflammation, is not evident in the majority of TAK patients. Most patients have no evident acute phase; the initial symptoms are manifestations caused by organ ischemia due to vessel stenosis or occlusion. Constitutional presentations, which are mainly due to inflammation, are found in more than one-third of patients in this study. This is 
Table 3 Manifestation of involved vessels in Takayasu's arteritis patients from different geographic areas

\begin{tabular}{|c|c|c|c|c|c|c|c|c|c|c|c|c|}
\hline \multirow[t]{2}{*}{ Arteries } & \multirow[t]{2}{*}{$\begin{array}{l}\text { This study } \\
(n=411)\end{array}$} & \multirow{2}{*}{$\begin{array}{l}\text { Mwipatayi } \\
\text { et al. [12] } \\
(n=272)\end{array}$} & \multicolumn{2}{|c|}{$\begin{array}{l}\text { Lee et al. } \\
\text { [22] }(n=204)\end{array}$} & \multicolumn{2}{|c|}{$\begin{array}{l}\text { Schmidt et al. } \\
\text { [21] }(n=126)\end{array}$} & \multicolumn{2}{|c|}{$\begin{array}{l}\text { Vanoli et al. } \\
\text { [24] }(n=104)\end{array}$} & \multicolumn{2}{|c|}{$\begin{array}{l}\text { Arnaud et al. } \\
\text { [23] }(n=82)\end{array}$} & \multicolumn{2}{|c|}{$\begin{array}{l}\text { Freitas et al. } \\
{[20](n=52)}\end{array}$} \\
\hline & & & Left & Right & Left & Right & Left & Right & Left & Right & Left & Right \\
\hline Subclavian artery & $328(79.8 \%)$ & ND & $67.1 \%$ & $55.2 \%$ & $66.3 \%$ & $41.0 \%$ & $65.6 \%$ & $52.5 \%$ & $68.3 \%$ & $50.0 \%$ & $60.6 \%$ & $40.4 \%$ \\
\hline Stenosis & 232 (56.4\%) & ND & $26.0 \%$ & $24.6 \%$ & $43.3 \%$ & $36.2 \%$ & $42.6 \%$ & $29.5 \%$ & ND & & $25.0 \%$ & $26.9 \%$ \\
\hline Occlusion & $130(31.6 \%)$ & ND & $34.8 \%$ & $14.3 \%$ & $29.8 \%$ & $4.8 \%$ & $23.0 \%$ & $23.0 \%$ & ND & & $25.0 \%$ & $5.8 \%$ \\
\hline Dilatation & $11(2.7 \%)$ & ND & $0.5 \%$ & $4.4 \%$ & ND & ND & 0 & 0 & ND & & 0 & $5.8 \%$ \\
\hline Aneurysm & $5(1.2 \%)$ & ND & & & 0 & $1.0 \%$ & $1.6 \%$ & 0 & ND & & $1.9 \%$ & $1.9 \%$ \\
\hline Carotid artery & 325 (79.1\%) & 83 (30.5\%) & $72.1 \%^{f}$ & $63.7 \%^{f}$ & $50.9 \%$ & $41.7 \%$ & $44.3 \%$ & $36.1 \%$ & $59.8 \%^{f}$ & $52.4 \%^{f}$ & $57.3 \%$ & $40.4 \%$ \\
\hline Stenosis/occlusion & $260(63.3 \%)$ & 59 (21.7\%) & ND & ND & ND & ND & ND & ND & ND & & ND & ND \\
\hline Stenosis & $241(58.6 \%)$ & ND & $33.3 \%^{f}$ & $32.3 \%^{f}$ & $41.7 \%$ & $37.0 \%$ & $37.7 \%$ & $23.0 \%$ & ND & & $30.8 \%$ & $23.1 \%$ \\
\hline Occlusion & $102(24.8 \%)$ & ND & $21.1 \%^{f}$ & $9.8 \%^{f}$ & $10.2 \%$ & $5.6 \%$ & $4.9 \%$ & $6.6 \%$ & ND & & $9.6 \%$ & $9.6 \%$ \\
\hline Dilatation & 16 (3.9\%) & ND & $1.0 \%^{f}$ & $1.5 \%^{f}$ & ND & ND & 0 & $3.3 \%$ & ND & & $1.9 \%$ & 0 \\
\hline Aneurysm & $2(0.5 \%)$ & 17 (6.3\%) & & & $0.9 \%$ & $0.9 \%$ & $1.6 \%$ & $3.3 \%$ & ND & & $5.8 \%$ & $1.9 \%$ \\
\hline $\begin{array}{l}\text { Aneurysm and } \\
\text { stenosis/occlusion }\end{array}$ & $1(0.2 \%)$ & 7 (2.6\%) & ND & ND & ND & ND & ND & ND & ND & & ND & ND \\
\hline Renal artery & 201 (48.9\%) & ND & $32.2 \%$ & $31.7 \%$ & $18.7 \%$ & $20.9 \%$ & $34.4 \%$ & $29.5 \%$ & $14.6 \%$ & $15.9 \%$ & $34.6 \%$ & $36.5 \%$ \\
\hline Stenosis & $182(44.3 \%)$ & ND & $25.2 \%$ & $25.2 \%$ & $16.5 \%$ & $19.8 \%$ & $31.2 \%$ & $23.0 \%$ & ND & & $15.4 \%$ & $25.0 \%$ \\
\hline Occlusion & $43(10.5 \%)$ & ND & $6.0 \%$ & $4.5 \%$ & $4.4 \%$ & $2.2 \%$ & $3.3 \%$ & $6.6 \%$ & ND & & $9.6 \%$ & $3.8 \%$ \\
\hline Dilatation & $6(1.5 \%)$ & ND & 0 & 0 & ND & ND & 0 & 0 & ND & & 0 & 0 \\
\hline Aneurysm & $2(0.5 \%)$ & ND & 0 & 0 & 0 & 0 & 0 & 0 & ND & & 0 & 0 \\
\hline Abdominal aorta & $158(38.4 \%)$ & $186(68.4 \%)$ & $63.2 \%$ & & $23.7 \%^{\mathrm{c}}$ & $27.4 \%^{d}$ & $39.3 \%$ & & $43.9 \%$ & & $63.5 \%$ & \\
\hline Stenosis/occlusion & $140(34.1 \%)$ & 115 (42.3\%) & ND & & ND & ND & ND & & ND & & ND & \\
\hline Stenosis & $134(32.6 \%)$ & ND & $38.3 \%$ & & $20.4 \%^{c}$ & $25.3 \%^{d}$ & $29.5 \%$ & & ND & & $26.9 \%$ & \\
\hline Occlusion & 12 (2.9\%) & ND & $4.0 \%$ & & $1.1 \%^{\mathrm{c}}$ & $2.1 \%^{d}$ & $6.6 \%$ & & ND & & $1.9 \%$ & \\
\hline Aneurysm & $6(1.4 \%)$ & $41(15.1 \%)$ & $6.0 \%$ & & $2.2 \%^{\mathrm{c}}$ & $1.1 \%^{\mathrm{d}}$ & $3.3 \%$ & & ND & & $7.7 \%$ & \\
\hline Dilatation & 0 & ND & & & ND & ND & ND & & ND & & $11.5 \%$ & \\
\hline $\begin{array}{l}\text { Aneurysm and } \\
\text { stenosis/occlusion }\end{array}$ & $4(1.0 \%)$ & $30(11.0 \%)$ & ND & & ND & ND & ND & & ND & & ND & \\
\hline Vertebral artery & $118(28.7 \%)$ & ND & ND & & $18.5 \%$ & $13.0 \%$ & $13.3 \%$ & $11.7 \%$ & $28.0 \%$ & $15.9 \%$ & $25.0 \%$ & $11.5 \%$ \\
\hline Stenosis & 82 (20.0\%) & ND & ND & & $15.7 \%$ & $9.3 \%$ & $8.3 \%$ & $8.3 \%$ & ND & & $13.5 \%$ & $5.8 \%$ \\
\hline Occlusion & 46 (11.2\%) & ND & ND & & $2.8 \%$ & $3.7 \%$ & $5.0 \%$ & $1.7 \%$ & ND & & $3.8 \%$ & $5.8 \%$ \\
\hline Dilatation & $10(2.4 \%)$ & ND & ND & & ND & ND & 0 & $1.7 \%$ & ND & & $1.9 \%$ & 0 \\
\hline Aneurysm & $1(0.2 \%)$ & ND & ND & & 0 & 0 & 0 & 0 & ND & & 0 & 0 \\
\hline Mesenteric artery & $116(29.7 \%)$ & $101(37.1 \%)$ & $22.8 \%^{\mathrm{a}}$ & $3.5 \%^{\mathrm{b}}$ & $24.7 \%^{a}$ & $6.9 \%^{\mathrm{b}}$ & $31.6 \%^{\mathrm{a}}$ & $9.4 \%^{\mathrm{b}}$ & ND & & $28.8 \%^{\mathrm{a}}$ & $3.8 \%^{\mathrm{b}}$ \\
\hline Stenosis/occlusion & $116(29.7 \%)$ & $92(33.8 \%)$ & ND & ND & ND & ND & ND & ND & ND & & ND & ND \\
\hline Stenosis & 90 (21.9\%) & ND & $14.4 \%^{\mathrm{a}}$ & $0.5 \%^{\mathrm{b}}$ & $18.0 \%^{\mathrm{a}}$ & $2.3 \%^{\mathrm{b}}$ & $24.6 \%^{\mathrm{a}}$ & $5.7 \%^{\mathrm{b}}$ & ND & & $15.4 \%^{\mathrm{a}}$ & $3.8 \%^{\mathrm{b}}$ \\
\hline Occlusion & $33(8.0 \%)$ & ND & $5.9 \%^{\mathrm{a}}$ & $2.0 \%^{\mathrm{b}}$ & $6.7 \%^{\mathrm{a}}$ & $4.6 \%^{b}$ & $7.0 \%^{\mathrm{a}}$ & $1.9 \%^{\mathrm{b}}$ & ND & & $11.5 \%^{\mathrm{a}}$ & $0^{b}$ \\
\hline Dilatation & $1(0.2 \%)$ & ND & $1.0 \%^{\mathrm{a}}$ & $1.0 \%^{\mathrm{b}}$ & ND & ND & $0^{\mathrm{a}}$ & $1.9 \%^{\mathrm{b}}$ & ND & & $0^{a}$ & $0^{b}$ \\
\hline Aneurysm & 0 & $9(3.3 \%)$ & & & $0^{\mathrm{a}}$ & $0^{b}$ & $0^{\mathrm{a}}$ & $0^{\mathrm{b}}$ & ND & & $0^{a}$ & $0^{b}$ \\
\hline Thoracic aorta & $86(17.5 \%)$ & $158(58.1 \%)$ & $57.2 \%$ & & $19.1 \%$ & & $11.1 \%$ & & $40.2 \%$ & & $34.6 \%$ & \\
\hline Stenosis/occlusion & $72(17.5 \%)$ & 98 (36.0\%) & ND & & $18.2 \%$ & & ND & & ND & & ND & \\
\hline Stenosis & $72(17.5 \%)$ & ND & $22.9 \%$ & & $18.2 \%$ & & $7.4 \%$ & & ND & & $9.6 \%$ & \\
\hline Occlusion & 0 & ND & 0 & & 0 & & 0 & & ND & & 0 & \\
\hline Dilatation & $14(3.4 \%)$ & ND & $3.0 \%$ & & ND & & $1.9 \%$ & & ND & & $9.6 \%$ & \\
\hline Aneurysm & $2(0.5 \%)$ & $38(14.0 \%)$ & & & $0.9 \%$ & & $1.9 \%$ & & ND & & $3.8 \%$ & \\
\hline $\begin{array}{l}\text { Aneurysm and } \\
\text { stenosis/occlusion }\end{array}$ & $1(0.2 \%)$ & 22 (8.1\%) & ND & & ND & & ND & & ND & & ND & \\
\hline
\end{tabular}


Table 3 Manifestation of involved vessels in Takayasu's arteritis patients from different geographic areas (Continued)

\begin{tabular}{|c|c|c|c|c|c|c|c|c|c|c|c|c|}
\hline Innominate artery & 81 (19.7\%) & $43(10.5 \%)$ & \multicolumn{2}{|l|}{$46.8 \%$} & \multicolumn{2}{|l|}{$25.5 \%$} & \multicolumn{2}{|l|}{$8.8 \%$} & \multicolumn{2}{|l|}{$28.0 \%$} & \multicolumn{2}{|l|}{$25.0 \%$} \\
\hline Stenosis/occlusion & 74 (18.0\%) & $22(8.1 \%)$ & \multicolumn{2}{|l|}{ ND } & \multicolumn{2}{|l|}{ ND } & \multicolumn{2}{|l|}{ ND } & \multicolumn{2}{|l|}{ ND } & \multicolumn{2}{|l|}{ ND } \\
\hline Stenosis & $61(14.8 \%)$ & ND & \multicolumn{2}{|l|}{$16.3 \%$} & \multicolumn{2}{|l|}{$18.9 \%$} & \multicolumn{2}{|l|}{$8.8 \%$} & \multicolumn{2}{|l|}{ ND } & \multicolumn{2}{|l|}{$11.5 \%$} \\
\hline Occlusion & $16(3.9 \%)$ & ND & \multicolumn{2}{|l|}{$3.0 \%$} & \multicolumn{2}{|l|}{$6.6 \%$} & \multicolumn{2}{|l|}{0} & \multicolumn{2}{|l|}{ ND } & \multicolumn{2}{|l|}{$1.9 \%$} \\
\hline Dilatation & $8(1.9 \%)$ & ND & \multicolumn{2}{|l|}{$4.0 \%$} & \multicolumn{2}{|l|}{ ND } & \multicolumn{2}{|l|}{$1.8 \%$} & \multicolumn{2}{|l|}{ ND } & \multicolumn{2}{|l|}{$7.7 \%$} \\
\hline Aneurysm & $1(0.2 \%)$ & 16 (5.9\%) & & & \multicolumn{2}{|l|}{$0.9 \%$} & 0 & & ND & & $1.9 \%$ & \\
\hline $\begin{array}{l}\text { Aneurysm and } \\
\text { stenosis/occlusion }\end{array}$ & 0 & $5(1.8 \%)$ & ND & & ND & & ND & & ND & & ND & \\
\hline Iliacofemoral artery & 65 (15.8\%) & $74(27.2 \%)$ & $13.3 \%^{9}$ & $14.8 \%^{\mathrm{g}}$ & $13.5 \%^{\mathrm{g}}$ & $13.5 \%^{\mathrm{g}}$ & $19.7 \%^{9}$ & $18.9 \%^{9}$ & $12.2 \%^{9}$ & $18.3 \%^{\mathrm{g}}$ & $23.1 \%^{9}$ & $23.1 \%^{9}$ \\
\hline Stenosis & 43 (10.5\%) & ND & $6.9 \%^{\mathrm{g}}$ & $8.9 \%^{9}$ & $10.1 \%^{9}$ & $10.1 \%^{9}$ & $9.8 \%^{g}$ & $6.6 \%^{9}$ & ND & & $11.5 \%^{\mathrm{g}}$ & $9.6 \%^{9}$ \\
\hline Occlusion & $18(4.4 \%)$ & ND & $4.9 \%^{9}$ & $3.4 \%^{9}$ & $4.5 \%^{9}$ & $2.2 \%^{9}$ & $8.2 \%^{9}$ & $9.8 \%^{9}$ & ND & & $5.8 \%^{9}$ & $5.8 \%^{9}$ \\
\hline Dilatation & 0 & ND & $0.5 \%^{9}$ & $0^{9}$ & ND & ND & $1.6 \%^{9}$ & $1.6 \%^{9}$ & ND & & $0^{g}$ & $0^{9}$ \\
\hline Aneurysm & $4(1.0 \%)$ & ND & & & $0^{9}$ & $2.2 \%^{9}$ & $0^{9}$ & $0^{9}$ & ND & & $0^{9}$ & $0^{9}$ \\
\hline Ascending aorta & 39 (9.5\%) & 65 (23.9\%) & $47.8 \%$ & & $9.1 \%$ & & ND & & ND & & $30.8 \%$ & \\
\hline Stenosis & $3(0.7 \%)$ & 15 (5.5\%) & 0 & & $2.7 \%$ & & ND & & ND & & $1.9 \%$ & \\
\hline Dilatation & 37 (9.0\%) & ND & $25.4 \%$ & & ND & & ND & & ND & & $19.2 \%$ & \\
\hline Aneurysm & $2(0.5 \%)$ & $43(15.8 \%)$ & & & $6.4 \%$ & & ND & & ND & & $3.8 \%$ & \\
\hline Aneurysm and stenosis & $1(0.2 \%)$ & $7(2.6 \%)$ & ND & & ND & & ND & & ND & & ND & \\
\hline Aortic arch & $32(7.8 \%)$ & 90 (33.1\%) & $37.9 \%$ & & $4.5 \%$ & & $10.3 \%$ & & $37.8 \%$ & & $19.2 \%$ & \\
\hline Stenosis & $25(6.1 \%)$ & $45(16.5 \%)$ & $0.5 \%$ & & $2.7 \%$ & & $5.2 \%$ & & ND & & $1.9 \%$ & \\
\hline Dilatation & $7(1.7 \%)$ & ND & $3.4 \%$ & & ND & & $5.2 \%$ & & ND & & $9.6 \%$ & \\
\hline Aneurysm & $1(0.2 \%)$ & $36(13.2 \%)$ & & & $1.8 \%$ & & 0 & & ND & & $1.9 \%$ & \\
\hline Aneurysm and stenosis & 0 & $9(3.3 \%)$ & ND & & ND & & ND & & ND & & ND & \\
\hline Pulmonary artery & $31 / 45^{\mathrm{e}}(68.9 \%)$ & $24(8.8 \%)$ & $13.4 \%$ & & $6 / 18^{\mathrm{e}}(33$ & $3 \%)$ & ND & & ND & & ND & \\
\hline Stenosis/occlusion & $26 / 45^{e}(57.8 \%)$ & $13(4.8 \%)$ & ND & & ND & & ND & & ND & & ND & \\
\hline Stenosis & $17 / 45^{\mathrm{e}}(37.8 \%)$ & ND & $4.0 \%$ & & $6 / 18^{\mathrm{e}}(33$ & $3 \%)$ & ND & & ND & & ND & \\
\hline Occlusion & $14 / 45^{\mathrm{e}}(31.1 \%)$ & ND & $2.0 \%$ & & $2 / 18^{\mathrm{e}}(11$ & $.1 \%)$ & ND & & ND & & ND & \\
\hline Dilatation & $1 / 45^{e}(2.2 \%)$ & ND & $2.0 \%$ & & ND & & ND & & ND & & ND & \\
\hline Aneurysm & 0 & $6(2.2 \%)$ & & & 0 & & ND & & ND & & ND & \\
\hline $\begin{array}{l}\text { Aneurysm and } \\
\text { stenosis/occlusion }\end{array}$ & 0 & $5(1.8 \%)$ & ND & & ND & & ND & & ND & & ND & \\
\hline Coronary artery & $15 / 42^{e}(35.7 \%)$ & ND & $31 / 49^{e}($ & 3.3\%) & $4 / 18^{e}(22$ & $2 \%)$ & ND & & ND & & ND & \\
\hline Stenosis & $14 / 42^{\mathrm{e}}(33.3 \%)$ & ND & $26 / 49^{e}$ & $3.1 \%)$ & $4 / 18^{\mathrm{e}}(22$ & $2 \%)$ & ND & & ND & & ND & \\
\hline Occlusion & $4 / 42^{\mathrm{e}}(9.5 \%)$ & ND & $2 / 49^{e}(4$ & & $1 / 18^{\mathrm{e}}(5$ & & ND & & ND & & ND & \\
\hline Dilatation & 0 & ND & $3 / 49^{e}(6$ & & ND & & ND & & ND & & ND & \\
\hline Aneurysm & 0 & ND & & & 0 & & ND & & ND & & ND & \\
\hline
\end{tabular}

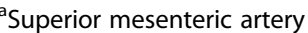

${ }^{b}$ Inferior mesenteric artery

'Suprarenal aorta

dInfrarenal aorta

${ }^{\mathrm{e}}$ Actually detected

fCommon carotid artery

giliac artery

$N D$ no data

parallel to the laboratory findings, including elevated level of ESR and hs-CRP (Table 1).

Through the literature review, we found that involvement of carotid arteries and subclavian arteries were similarly high in the seven studies [12, 20-24], including the present one (Table 3). It is widely accepted that carotid arteries are the most commonly involved arteries in TAK patients. The symptoms and findings from physical examination of carotid arteries are usually obvious and lead to the consideration of the diagnosis of TAK. 
Table 4 Angiographic classification of Takayasu's arteritis patients from different populations

\begin{tabular}{llllllllllll}
\hline & China/this study & Turkey [16] & Korea [22] & US [21] & China [15] & Mexico [34] & Korea [25] & India [26] & Japan [26] & Thailand [13] \\
\hline$n$ & 411 & 248 & $204^{\mathrm{d}}$ & $126^{\mathrm{e}}$ & 125 & 110 & 108 & 102 & 79 & 63 \\
Disease durations & 21.0 & 34.2 & $\mathrm{ND}$ & 13.3 & 19 & $\mathrm{ND}$ & $13.9 \pm 10.1$ & ND & $24.3 \pm 11.5^{\mathrm{c}}$ & ND & \\
(months) & $(6.0,60.0)^{\mathrm{a}}$ & $(0,240)^{\mathrm{b}}$ & & $(6.1,35.5)^{\mathrm{a}}$ & $(0.5,160)^{\mathrm{b}}$ & & $(1,60)^{\mathrm{b}}$ & & & \\
Type I (\%) & 22.1 & 32 & 11.1 & 20 & 40 & 19 & 36.1 & 6.9 & 24.1 & 0 \\
Type IIa (\%) & 3.9 & 6.9 & 8.6 & 6 & 4.8 & 3 & 2.8 & 1 & 11.4 & 0 \\
Type IIb (\%) & 3.9 & 3.2 & 14.1 & 7 & 1.6 & 4 & 4.6 & 5.9 & 10.1 & 11.1 \\
Type III (\%) & 2.9 & 3.2 & 4.0 & 5 & 2.4 & 4 & 7.4 & 2.9 & 0 & 3.2 \\
Type IV (\%) & 6.3 & 3.7 & 7.6 & 5 & 20.8 & 2 & 15.7 & 28.4 & 1.3 & 19 \\
Type V (\%) & 60.8 & 51 & 54.5 & 57 & 30.4 & 69 & 33.3 & 54.9 & 53.2 & 66.7 &
\end{tabular}

${ }^{a}$ Median $(\mathrm{Q} 1, \mathrm{Q} 3)$

${ }^{\mathrm{b}}$ Median (range)

${ }^{\mathrm{C}}$ Years

${ }^{d} 198$ patients had undergone angiographic evaluation

${ }^{\mathrm{e}} 100$ patients had undergone angiographic evaluation

ND no data

But the symptoms of subclavian arteries are insidious until severe ischemia develops, presenting as claudication of the upper limbs, imbalance of pulsation, or differences in bilateral blood pressure. The lesions of subclavian arteries are occasionally missed during screening of TAK-related arterial presentation by Doppler ultrasound because of the technical difficulties. Because of the high occurrence rate of subclavian arterial lesions in TAK, we suggest that careful screening of subclavian arterial lesions should be done, especially when patients present with symptoms or signs suggesting subclavian arterial ischemia.

In general, the major angiographic features of TAK are artery stenosis and occlusion, with relatively few dilatations and aneurysms. However, in the study by Mwipatayi and co-researchers [12], 305 aneurysms were found in 272 TAK patients. However, in Chinese cohort studies, the reported occurrence of aneurysm ranged from $3.4 \%$ to $4.8 \%$ [14, 15]. Even in different areas of Asia, the occurrence rate of aneurysms varied markedly. In a cohort study from Thailand [13], 38 aneurysms were found in 63 TAK patients. In the Chinese cohort reported by Cong et al. [15], 6 aneurysms were found in 125 TAK patients. In the present study, 33 (8.0\%) aneurysms were found in 411 Chinese TAK patients (Table 2), which is similar to the study of Cong et al. but much lower than in the study of Mwipatayi et al. or the Thai study $[12,13]$. This may suggest that genetic factors might play a role in the pathogenesis of TAK. The high prevalence of TAK in Asia may be related to environmental factors, such as the prevalence of tuberculosis infection [30], which was assumed to be associated with TAK pathogenesis and development [31], while no direct evidence was found in arterial lesions of TAK patients [32, 33].
We compared the angiographic classification pattern of our patients with nine groups of TAK patients from different geographic areas in eight reported studies $[13,15,16,22,23,25,26,34]$ (Table 4). The most common pattern in seven of the studies was type $\mathrm{V}$, as in this study, while in the other two groups it was type I. This difference might result from the selection of patients at different stages of TAK development. Since TAK is a chronic inflammatory disease, patients are prone to have the type I pattern in the early stage of TAK, while type $\mathrm{V}$ may be seen in the late stage. Autopsy in Japanese TAK patients showed evidence that various stages of inflammation existed in the aortic walls, including infiltration of inflammatory cells, granulomatosis, and fibrosis [35]. The new active lesions were usually found adjacent to the fibrotic ones [35], which suggested consecutive inflammatory progression in the vessel wall in the involved arteritis. Therefore, a longer disease duration was associated with more extended lesions in TAK. As the disease duration of our patients was usually long, this may be the reason that type $\mathrm{V}$ was the most common pattern in this study.

In this study, bypass surgery $(54 / 411,13.1 \%)$ was the most common surgical intervention for TAK-related lesions, followed by PTA $(25 / 411,6.1 \%)$ to relieve refractory hypertension caused by severe renal artery stenosis (Table 3). This is consistent with the distribution pattern of involved vessels in this group of patients. Stenosis of carotid arteries, subclavian arteries, and renal arteries was found in $58.6 \%, 56.4 \%$, and $44.3 \%$ of patients, respectively, which were the three most commonly involved arteries in this study (Table 2). Dizziness or upper limb intermittent claudication is the most prominent clinical manifestation of stenosis of cervical vessels, which may be the initial clinical presentation 
Table 5 The surgical and interventional operations taken in 411 Chinese Takayasu's patients

\begin{tabular}{|c|c|c|}
\hline Category of operations/interventions & Patient numbers & $\%$ \\
\hline Operations on cervical vessels & 53 & 12.9 \\
\hline Bypass between aorta and carotid artery & 21 & 5.1 \\
\hline Bypass between carotid and subclavian arteries & 11 & 2.7 \\
\hline Bypass between aorta and subclavian arteries & 6 & 1.5 \\
\hline Angioplasty and/or stenting in carotid arteries & 6 & 1.5 \\
\hline Angioplasty and/or stenting in subclavian arteries & 4 & 1.0 \\
\hline Angioplasty and/or stenting in innominate arteries & 3 & 0.7 \\
\hline Bypass between innominate and carotid arteries & 1 & 0.2 \\
\hline Bypass between innominate and subclavian arteries & 1 & 0.2 \\
\hline Operations to reduce renal hypertension & 33 & 8.0 \\
\hline Angioplasty and/or stenting in renal arteries & 25 & 6.1 \\
\hline Bypass between aorta and renal arteries & 4 & 1.0 \\
\hline Nephrectomy & 4 & 1.0 \\
\hline Operations on aortic and iliacofemoral stenosis or occlusion & 20 & 4.9 \\
\hline Angioplasty and/or stenting in aorta & 12 & 2.9 \\
\hline Aorta bypass grafting & 5 & 1.2 \\
\hline Bypass between aorta and bilateral iliac arteries & 1 & 0.2 \\
\hline Bypass between aorta and bilateral femoral arteries & 1 & 0.2 \\
\hline Angioplasty and/or stenting in common iliac arteries & 1 & 0.2 \\
\hline Operations on valvar lesions and coronary artery & 9 & 2.2 \\
\hline Cardiac valve replacement & 5 & 1.2 \\
\hline Angioplasty and/or stenting in coronary arteries & 3 & 0.7 \\
\hline Coronary artery bypass grafting & 1 & 0.2 \\
\hline Repair on aortic aneurysm & 2 & 0.5 \\
\hline Ascending aorta and aortic arch & 1 & 0.2 \\
\hline Thoracic and abdominal aorta & 1 & 0.2 \\
\hline Rebuilding circulation in upper limbs & 1 & 0.2 \\
\hline Bypass between axillary arteries & 1 & 0.2 \\
\hline Rebuilding circulation in mesenteric arteries & 1 & 0.2 \\
\hline Mesenteric artery bypass & 1 & 0.2 \\
\hline \multicolumn{3}{|l|}{ Summary } \\
\hline Bypass operations & 54 & 13.1 \\
\hline Percutaneous transluminal angiography (PTA) & 47 & 11.4 \\
\hline PTA in renal arteries & 25 & 6.1 \\
\hline Stenting & 35 & 8.5 \\
\hline Other operations & 11 & 2.7 \\
\hline
\end{tabular}

that drives patients to seek medical support. However, the leading causes for surgical operations were renovascular hypertension $(48 / 155,31.0 \%)$, hypertension due to aortic coarctation $(32 / 155,20.6 \%)$, and carotid arterial occlusion $(26 / 155,16.8 \%)$ in the report by Miyata et al. [36]. The difference in the occurrence rates of carotid stenosis lesion and aortic coarctation in different ethnic groups would lead to the difference in the rate of surgical intervention for TAK patients.
The most common cause of death in this study was heart failure, which was secondary to hypertension and aortic regurgitation [29]. In this study, which included TAK patients admitted to PUMCH over the past 24 years, the main direct cause of death was heart failure (4/12, $33.3 \%)$, followed by hemorrhagic shock $(2 / 12,16.7 \%)$ due to gastrointestinal tract bleeding, and septicemia secondary to pulmonary infection $(2 / 12,16.7 \%)$. Heart failure was secondary to hypertension and aortic regurgitation in 
most of the deceased patients. These suggest that controlling blood pressure to the normal range may be important in preventing the death of TAK patients. The hemorrhagic complications might be related to the use of low-dose aspirin (75 or $100 \mathrm{mg} /$ day) in TAK patients; however, de Souza and co-researchers have proven that antiplatelet therapy was associated with a lower frequency of ischemic events in patients with TAK [37]. Thus they suggested that the use of antiplatelet therapy in TAK patients was more beneficial than harmful.

The major limitation of this study is that only inpatients were included. Therefore, the selection of the study patients was biased. The retrospective analysis and chart review placed the study at the risk of recall bias, which may compromise the power of the conclusion. There was also a lack of standardized protocol to evaluate the arterial lesions. Further prospective longitudinal study is needed.

\section{Conclusions}

In conclusion, blood vessel involvement in Chinese TAK patients is different from other ethnic groups. Aortic aneurysm is less common in Chinese TAK patients, while the subclavian artery and carotid artery may be more commonly involved in Chinese TAK patients. In addition, when compared with other studies, type V is more common in Chinese TAK patients. The difference in angiographic features may lead to the difference in clinical manifestations, and also the difference in surgical interventions. The most common cause of death in Chinese TAK patients in this study is heart failure secondary to hypertension caused by renal artery involvement, which suggests that more attention should be paid to controlling blood pressure in the normal range.

\section{Abbreviations \\ CRP: C-reactive protein; CTA: Computed tomography angiography; ESR: Erythrocyte sedimentation rate; hs-CRP: High-sensitive C-reactive protein; MRA: Magnetic resonance angiography; PTA: Percutaneous transluminal angiography; TAK: Takayasu's arteritis}

\section{Acknowledgements}

Not applicable.

\section{Funding}

None.

\section{Availability of data and materials}

The datasets used and/or analyzed during the current study are available from the corresponding author on reasonable request.

\section{Authors' contributions}

$X T$ and $X Z$ conceived and designed the study. ML advised on the design of the study. JL, FS, ZC, and YY collected, entered, and cross-checked the data. $X T$ and $J \mathrm{~L}$ reviewed and re-checked the diagnosis of all patients. $J \mathrm{~L}$ and $J \mathrm{Z}$ analyzed the data. $J \mathrm{~L}$ drafted the paper. $J \mathrm{~L}, \mathrm{XT}$, and $\mathrm{XZ}$ edited and revised the paper. All authors have read and approved the final manuscript.

\section{Competing interests}

The authors declare that they have no competing interests.
Consent for publication

Not applicable.

Ethics approval and consent to participate

The study protocol was approved by the Institutional Review Board of Peking Union Medical College Hospital. Written informed consent was waived due to the retrospective nature of this study.

\section{Publisher's Note}

Springer Nature remains neutral with regard to jurisdictional claims in published maps and institutional affiliations.

Received: 9 December 2016 Accepted: 2 May 2017

Published online: 25 May 2017

\section{References}

1. Matsubara O, Kuwata T, Nemoto T, et al. Coronary artery lesions in Takayasu arteritis: pathological considerations. Heart Vessels Suppl. 1992;7:26-31.

2. Reinhold-Keller E, Herlyn K, Wagner-Bastmeyer R, et al. Stable incidence of primary systemic vasculitides over five years: results from the German vasculitis register. Arthritis Rheum. 2005;53(1):93-9.

3. Gudbrandsson B, Molberg O, Garen T, et al. Prevalence, Incidence, and Disease Characteristics of Takayasu Arteritis by Ethnic Background: Data From a Large, Population-Based Cohort Resident in Southern Norway. Arthritis Care Res. 2017;69(2):278-85.

4. Saritas F, Donmez S, Direskeneli $\mathrm{H}$, et al. The epidemiology of Takayasu arteritis: a hospital-based study from northwestern part of Turkey. Rheumatol Int. 2016;36(7):911-6.

5. Watts R, Al-Taiar A, Mooney J, et al. The epidemiology of Takayasu arteritis in the UK. Rheumatology (Oxford). 2009;48(8):1008-11.

6. Toshihiko N. Current status of large and small vessel vasculitis in Japan. Int J Cardiol. 1996;54(Suppl):S91-8.

7. Nasu T. Takayasu's truncoarteritis in Japan. A statistical observation of 76 autopsy cases. Pathol Microbiol. 1975;43(2-0):140-6.

8. Dreyer L, Faurschou M, Baslund B. A population-based study of Takayasu s arteritis in eastern Denmark. Clin Exp Rheumatol. 2011;29(1 Suppl 64):S40-2.

9. Waern AU, Andersson P, Hemmingsson A. Takayasu's arteritis: a hospitalregion based study on occurrence, treatment and prognosis. Angiology. 1983;34(5):311-20

10. el-Reshaid K, Varro J, al-Duwairi Q. Takayasu's arteritis in Kuwait. Am J Trop Med Hyg. 1995;98(5):299-305.

11. Soto ME, Espinola-Zavaleta N, Ramirez-Quito O, et al. Echocardiographic follow-up of patients with Takayasu's arteritis: five-year survival. Echocardiography. 2006;23(5):353-60.

12. Mwipatayi $B P$, Jeffery $P C$, Beningfield SJ, et al. Takayasu arteritis: clinical features and management: report of 272 cases. ANZ J Surg. 2005;75(3):110-7.

13. Suwanwela N, Piyachon C. Takayasu arteritis in Thailand: clinical and imaging features. Int J Cardiol. 1996;54(Suppl):S117-34.

14. Zheng D, Fan D, Liu L. Takayasu arteritis in China: a report of 530 cases. Heart Vessels Suppl. 1992;7:32-6.

15. Cong XL, Dai SM, Feng X, et al. Takayasu's arteritis: clinical features and outcomes of 125 patients in China. Clin Rheumatol. 2010;29(9):973-81.

16. Bicakcigil M, Aksu K, Kamali S, et al. Takayasu's arteritis in Turkey-clinical and angiographic features of 248 patients. Clin Exp Rheumatol. 2009; 27(1 Suppl 52):S59-64.

17. Arend WP, Michel BA, Bloch DA, et al. The American College of Rheumatology 1990 criteria for the classification of Takayasu arteritis. Arthritis Rheum. 1990:33(8):1129-34.

18. Writing Committee M, Yancy CW, Jessup M, et al. 2013 ACCF/AHA guideline for the management of heart failure: a report of the American College of Cardiology Foundation/American Heart Association Task Force on practice guidelines. Circulation. 2013;128(16):e240-327

19. Hata A, Noda M, Moriwaki R, et al. Angiographic findings of Takayasu arteritis: new classification. Int J Cardiol. 1996;54(Suppl):S155-63.

20. Freitas DS, Camargo CZ, Mariz HA, et al. Takayasu arteritis: assessment of response to medical therapy based on clinical activity criteria and imaging techniques. Rheumatol Int. 2012;32(3):703-9.

21. Schmidt J, Kermani TA, Bacani AK, et al. Diagnostic features, treatment, and outcomes of Takayasu arteritis in a US cohort of 126 patients. Mayo Clin Proc. 2013;88(8):822-30. 
22. Lee GY, Jang SY, Ko SM, et al. Cardiovascular manifestations of Takayasu arteritis and their relationship to the disease activity: analysis of 204 Korean patients at a single center. Int J Cardiol. 2012;159(1):14-20.

23. Arnaud L, Haroche J, Toledano D, et al. Cluster analysis of arterial involvement in Takayasu arteritis reveals symmetric extension of the lesions in paired arterial beds. Arthritis Rheum. 2011;63(4):1136-40.

24. Vanoli M, Daina E, Salvarani C, et al. Takayasu's arteritis: a study of 104 Italian patients. Arthritis Rheum. 2005;53(1):100-7.

25. Park MC, Lee SW, Park YB, et al. Clinical characteristics and outcomes of Takayasu's arteritis: analysis of 108 patients using standardized criteria for diagnosis, activity assessment, and angiographic classification. Scand J Rheumatol. 2005;34(4):284-92.

26. Moriwaki R, Noda M, Yajima M, et al. Clinical manifestations of Takayasu arteritis in India and Japan-new classification of angiographic findings. Angiology. 1997:48(5):369-79.

27. Canas CA, Jimenez CA, Ramirez LA, et al. Takayasu arteritis in Colombia. Int J Cardiol. 1998;66 Suppl 1:S73-9.

28. Sato El, Lima DN, Espirito Santo B, et al. Takayasu arteritis. Treatment and prognosis in a university center in Brazil. Int J Cardiol. 2000:75 Suppl 1:S163-6.

29. Li J, Zhu M, Li M, et al. Cause of death in Chinese Takayasu arteritis patients. Medicine (Baltimore). 2016;95(27):e4069.

30. World Health Organisation. Global Tuberculosis report. 2014

31. Arnaud L, Haroche J, Mathian A, et al. Pathogenesis of Takayasu's arteritis: a 2011 update. Autoimmun Rev. 2011;11(1):61-7.

32. Carvalho ES, de Souza AW, Leao SC, et al. Absence of mycobacterial DNA in peripheral blood and artery specimens in patients with Takayasu arteritis. Clin Rheumatol. 2017;36(1):205-8.

33. Arnaud L, Cambau E, Brocheriou I, et al. Absence of mycobacterium tuberculosis in arterial lesions from patients with Takayasu's arteritis. J Rheumatol. 2009;36(8):1682-5.

34. Soto ME, Espinola N, Flores-Suarez LF, et al. Takayasu arteritis: clinical features in 110 Mexican Mestizo patients and cardiovascular impact on survival and prognosis. Clin Exp Rheumatol. 2008;26(3 Suppl 49):S9-15.

35. Hotchi M. Pathological studies on Takayasu arteritis. Heart Vessels Suppl. 1992;7:11-7.

36. Miyata $\mathrm{T}$, Sato $\mathrm{O}$, Koyama $\mathrm{H}$, et al. Long-term survival after surgical treatment of patients with Takayasu's arteritis. Circulation. 2003;108(12):1474-80.

37. de Souza AW, Machado NP, Pereira VM, et al. Antiplatelet therapy for the prevention of arterial ischemic events in takayasu arteritis. Circ J. 2010;74(6):1236-41.

\section{Submit your next manuscript to BioMed Central and we will help you at every step:}

- We accept pre-submission inquiries

- Our selector tool helps you to find the most relevant journal

- We provide round the clock customer support

- Convenient online submission

- Thorough peer review

- Inclusion in PubMed and all major indexing services

- Maximum visibility for your research

Submit your manuscript at www.biomedcentral.com/submit

) Biomed Central 